\title{
Supporting Information for Solution Deposition of Self-Assembled Benzoate Monolayers on Rutile (110): Effect of $\pi-\pi$ Interactions on Monolayer Structure
}

Erik S. Skibinski, Anqi Song, William J. I. DeBenedetti, Amnon G. Ortoll-Bloch, and Melissa A. Hines* Dept. of Chemistry and Chemical Biology, Cornell University, Ithaca NY 14853 USA

\section{XPS Quantification}

The fractional coverage $\phi$ of the benzoate layer on a rutile (110) surface was calculated from the integrated areas of the $\mathrm{C} 1 s$ and Ti $2 p_{3 / 2}$ XPS transitions, $I_{\mathrm{C}}$ and $I_{\mathrm{Ti}}$, using the geometry of the benzoate monolayer and the Beer-Lambert law. Starting from the DFT-calculated structure and neglecting scattering by the $\mathrm{H}$ atoms, the benzoate layer was modeled as a uniform, 0.50 -nm-thick layer of $\mathrm{C}$ atoms with a density $20 \%$ of that of graphite (i.e., the calculated density). As a result of the reduced density, the mean free paths of photoelectrons in the monolayer were assumed to be 5 times greater than in dense materials. The $\mathrm{C}$ and the $\mathrm{Ti}$ atoms emitted photoelectrons at an angle $\theta$ from the surface normal; however, some of the photoelectrons scattered within the sample. As a result, the relative areas of the two bands were given by

$$
\frac{I_{C}}{I_{T i}}=\frac{0.20}{0.333} \frac{\Gamma_{C}}{\Gamma_{T i}} \frac{\phi \int_{0}^{a} e^{-x / 5 \lambda_{c} \cos \theta} d x}{\int_{0}^{\infty} e^{-a / 5 \lambda_{n} \cos \theta} e^{-x / \lambda_{n} \cos \theta} d x}
$$

where $a=0.50 \mathrm{~nm}, \Gamma_{\mathrm{C}}$ and $\Gamma_{\mathrm{Ti}}$ are the relative photoemission cross-sections of the $\mathrm{C}$ and $\mathrm{Ti}$ atoms, respectively, and $\lambda_{\mathrm{C}}$ and $\lambda_{\mathrm{Ti}}$ are the mean free paths of the $\mathrm{C}$ and Ti photoelectrons. The factor of $1 / 3$ in the denominator accounts for the stoichiometry of $\mathrm{Ti}$ in $\mathrm{TiO}_{2}$. Since the monolayer thickness was much smaller than 5 mean free paths, the integrals could be performed and the results expanded to first order in $a / 5 \lambda_{C} \cos \theta$ yielding

$$
\frac{I_{C}}{I_{T i}}=0.60 \frac{\Gamma_{C}}{\Gamma_{T i}} \frac{\phi a}{e^{-a / 5 \lambda_{i} \cos \theta} \lambda_{T i} \cos \theta} .
$$

Using similar reasoning, the relative photoelectron intensities from bulk samples of graphite and Ti were

$$
\frac{I_{C}^{\infty}}{I_{T i}^{\infty}}=\frac{\Gamma_{C}}{\Gamma_{T i}} \frac{\int_{0}^{\infty} e^{-x / \lambda_{c} \cos \theta} d x}{\int_{0}^{\infty} e^{-x / \lambda_{n} \cos \theta} d x}=\frac{\Gamma_{C}}{\Gamma_{T i}} \frac{\lambda_{C}}{\lambda_{T i}}=\frac{R S F_{C}}{R S F_{T i}}
$$

where $\mathrm{RSF}_{X}$ is the relative sensitivity factor of species $X$, a quantity tabulated by Wagner, et al. ${ }^{1}$ Solving for the monolayer coverage yields

$$
\begin{aligned}
\phi & =1.7 \frac{R S F_{T i}}{R S F_{C}} e^{-a / 5 \lambda_{n} \cos \theta} \lambda_{C} \cos \theta \frac{I_{C}}{I_{T i}} \\
& =13 e^{-0.079 / \cos \theta} \cos \theta \frac{I_{C}}{I_{T i}}
\end{aligned}
$$

In simplifying Eqn. (4), the mean free paths of the $\mathrm{C}$ and $\mathrm{Ti}$ photoelectrons in $\mathrm{TiO}_{2}$ were interpolated from Fuentes, et al., ${ }^{2}$ which yielded $\lambda_{\mathrm{C}}=1.54 \mathrm{~nm}$ and $\lambda_{\mathrm{Ti}}=1.27 \mathrm{~nm}$, and the ratio of relative sensitivity factor, $\mathrm{RSF}_{\mathrm{Ti}} / \mathrm{RSF}_{\mathrm{C}}$, was 4.8 from the data of Wagner, et al. ${ }^{1}$ 


\section{Vibrational Energy Calculations}

To make efficient use of computational resources, vibrational energies were calculated using density functional perturbation theory without dispersion interactions on a $4 \times 2$, 2-trilayer-thick periodically repeating slab as shown in Figure S 1 . Because of the reduced thickness of the slab, broken Ti and O bonds on the bottom of the slab were terminated by artificial atoms (pseudohydrogen) with $+4 / 3$ and $+2 / 3$ nuclear charges, respectively. ${ }^{3,4}$ The atoms in the slab were held in the equilibrium geometry determined from similar calculations on 5-trilayer-thick slabs. Only the gamma point was included in the calculation. The 208 vibrational degrees of freedom included motion of all atoms above the uppermost Ti-O plane.

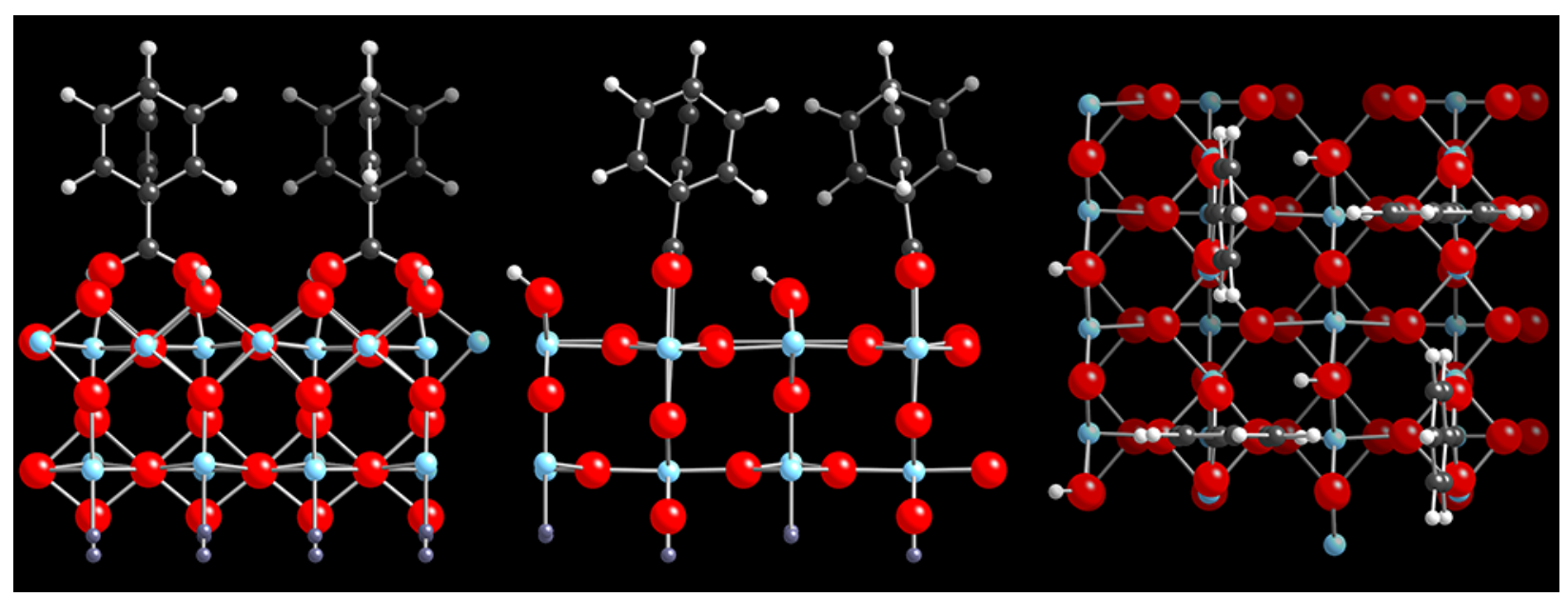

Figure S 1: Structure of the relaxed supercell used to calculate the infrared spectrum of the benzoate monolayer. The Ti, O, C, H, and pseudo-H atoms are light blue, red, black, white, and gray, respectively. The views are from the side along the (left) [1 $\overline{10}]$ and (middle) [001] directions, and from the (right) top.

The transition dipole moment $\vec{\mu}_{l}$ of the $l^{\text {th }}$ eigenmode was calculated from the Born effective charge tensor $\vec{Z}_{n}^{*}$ and normalized displacements $\vec{d}_{l}$ of the $n$ atoms using

$$
\vec{\mu}_{l}=\sum_{n} \vec{Z}_{n}^{*} \vec{d}_{n, l} .
$$

The total infrared absorbance $A_{\text {total }}$ of the system is given by

$$
A_{\text {total }}(\omega)=\sum_{l} \int I\left(\omega-\omega_{0}\right)\left|\mu_{l}\right|^{2} \delta\left(\omega_{0}-\omega_{l}\right) d \omega_{0},
$$

where $\omega_{1}$ is the energy of the $l^{\text {th }}$ normal mode, and $I$ is the spectral lineshape function (here, Gaussian). If light polarized along $\hat{j}$ is used to collect the infrared spectrum, the " $j$-polarized absorbance" $A_{\mathrm{j}}$ is given by

$$
A_{j}(\omega)=\sum_{l} \int I\left(\omega-\omega_{0}\right)\left(\hat{j} \cdot \vec{\mu}_{l}\right)^{2} \delta\left(\omega_{0}-\omega_{l}\right) d \omega_{0} .
$$

Importantly, we note that these calculated absorbances cannot be directly compared to spectra obtained with either unpolarized or $p$-polarized radiation. ${ }^{5,6}$ The $z$-component of the electric field at the surface is screened by an unknown surface dielectric constant that cannot be estimated. For example on $\mathrm{Si}$ substrates, this effect reduces the intensity of $z$-polarized modes by a factor of $\sim 4$. Spectra obtained with $p$-polarized radiation contain contributions from in-plane and $z$-oriented modes; however, the relative contributions of these modes cannot be estimated without knowledge of the surface dielectric constant. 
Figure S 2 compares the experimental and calculated spectrum of the benzoate monolayer under different polarization conditions. Spectra taken with $s$-polarized radiation should be directly comparable to the calculated in-plane-polarized spectra. The experimental and calculated mode energies are in good agreement; however, the mode structures are different. The origins of these discrepancies are unclear. We note that there are few benchmarking studies on surface vibrational modes. Infrared absorption probes the second derivative of the potential energy with respect to displacement, so these discrepancies may be related to the approximations inherent to the PBE functional or to approximations necessitated by the large system size.

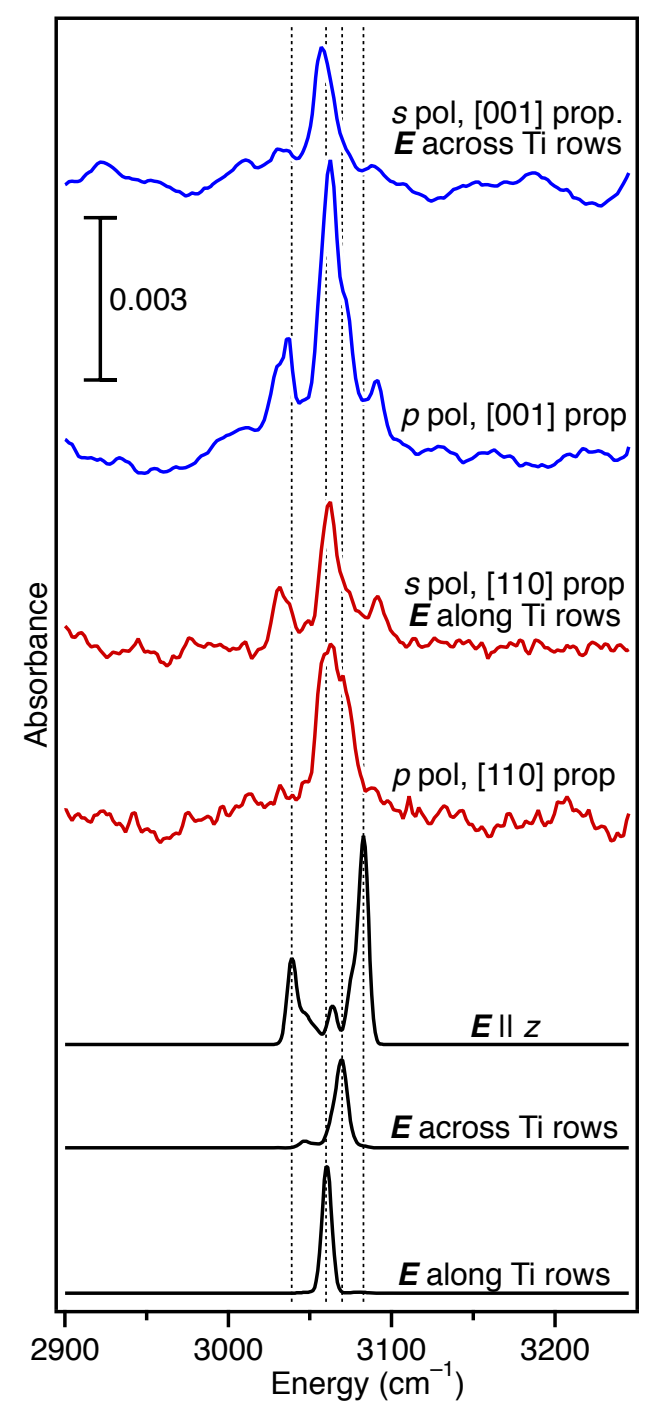

Figure S 2: A comparison of the $\mathrm{C}-\mathrm{H}$ stretch region of the (red, blue) experimental and (black) calculated infrared spectrum of the benzoate monolayer taken with light in different incident directions and polarizations. The Gaussian linewidth and absorbance scale factor for the calculated spectra were chosen arbitrarily.

\section{Structures used in DFT Calculations}

Structures were modeled using $4 \times 2$ periodically repeating slabs, where the first and second integers refer to the number of repeat units in the [001] and [1 $\overline{1} 0]$ directions, respectively, consisting of $5 \mathrm{TiO}_{2}$ trilayers separated by a $12.5 \AA$ vacuum spacing. Different surface and subsurface positions for the dissociated $\mathrm{H}$ atom were tested; only the minimum energy structures are reported. 
The slab used to model the lowest energy structure of a benzoate monolayer, the paired tetramer configuration, is shown in Figure S 3. Three different dimer structures were modeled in both vertical and tilted configurations: a parallel dimer, a $\mathrm{T}$ dimer, and a staggered parallel dimer. The tilted structures are shown in Figure S 4-Figure S 6.

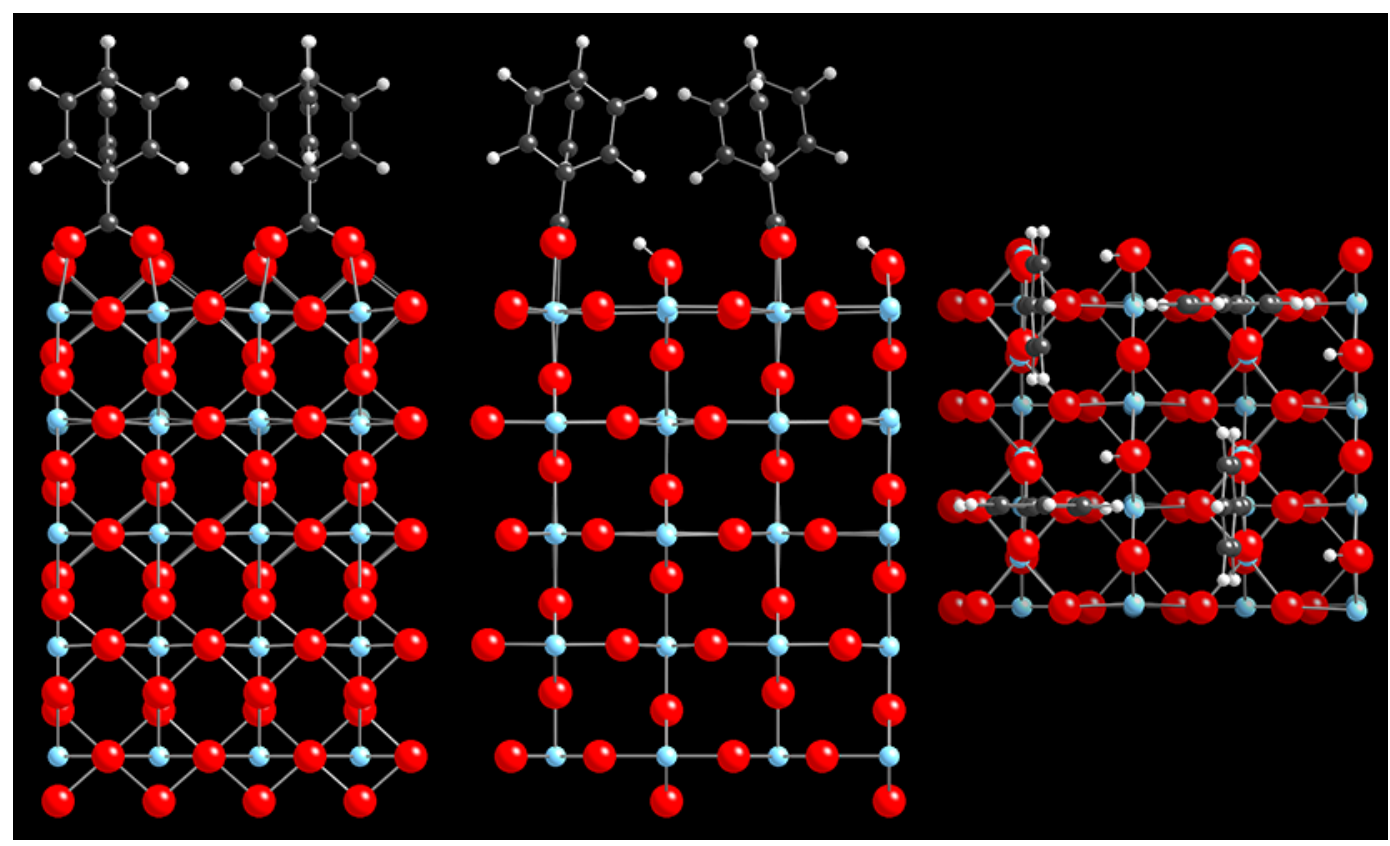

Figure S 3: Structure of the relaxed supercell used to represent the lowest energy structure of a benzoate monolayer. The benzoate monomers are arranged in a tetramer in which every phenyl ring is oriented in an edge-to-face geometry with its four nearest neighbors. Adjacent pairs of benzoate molecules tilt toward one another by $7^{\circ}$ along the [1 $1 \overline{1} 0]$ direction. 


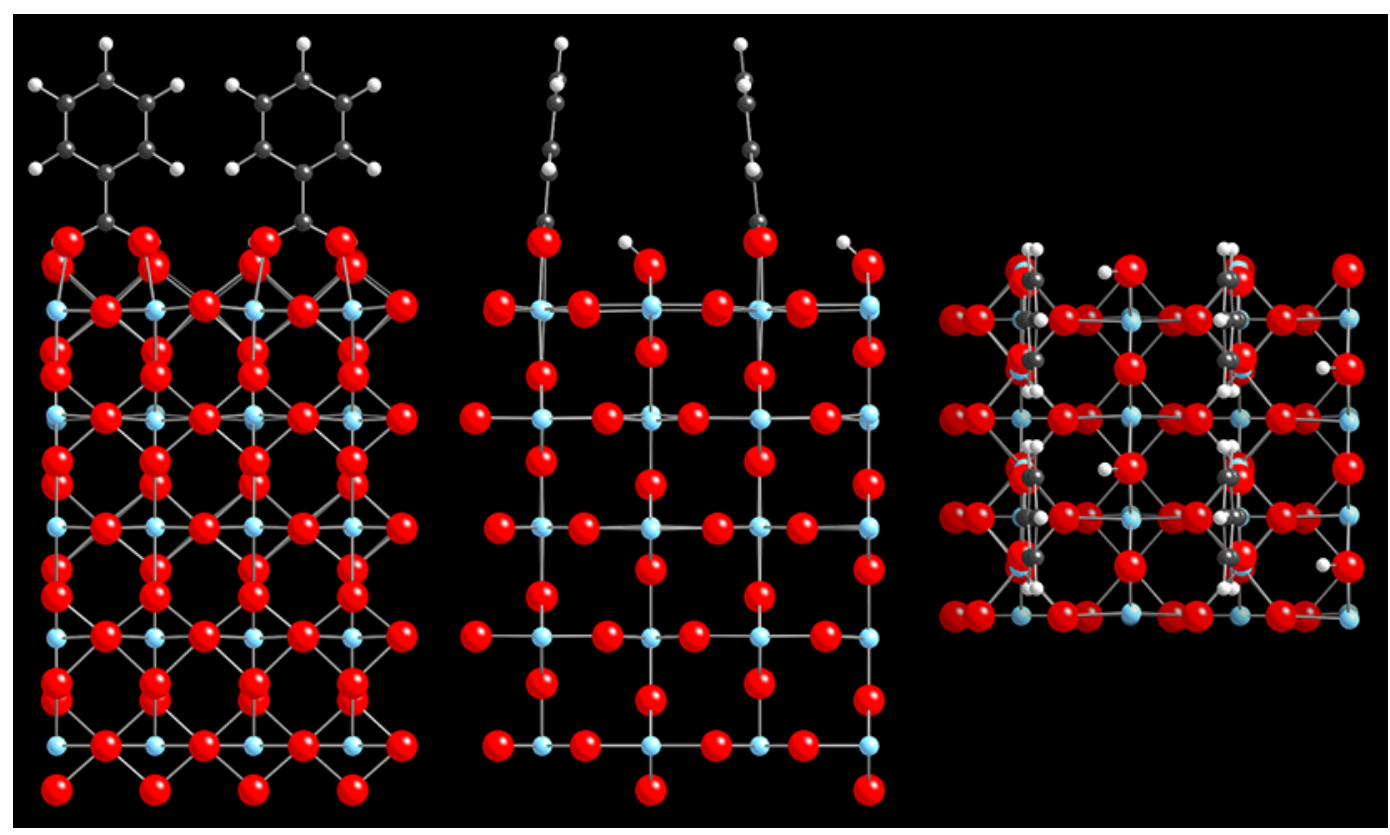

Figure S 4: Structure of the relaxed supercell used to represent the tilted parallel benzoate dimer. The views are from the side along the (left) [1 $\overline{10}$ ] and (middle) [001] directions, and from the (right) top.

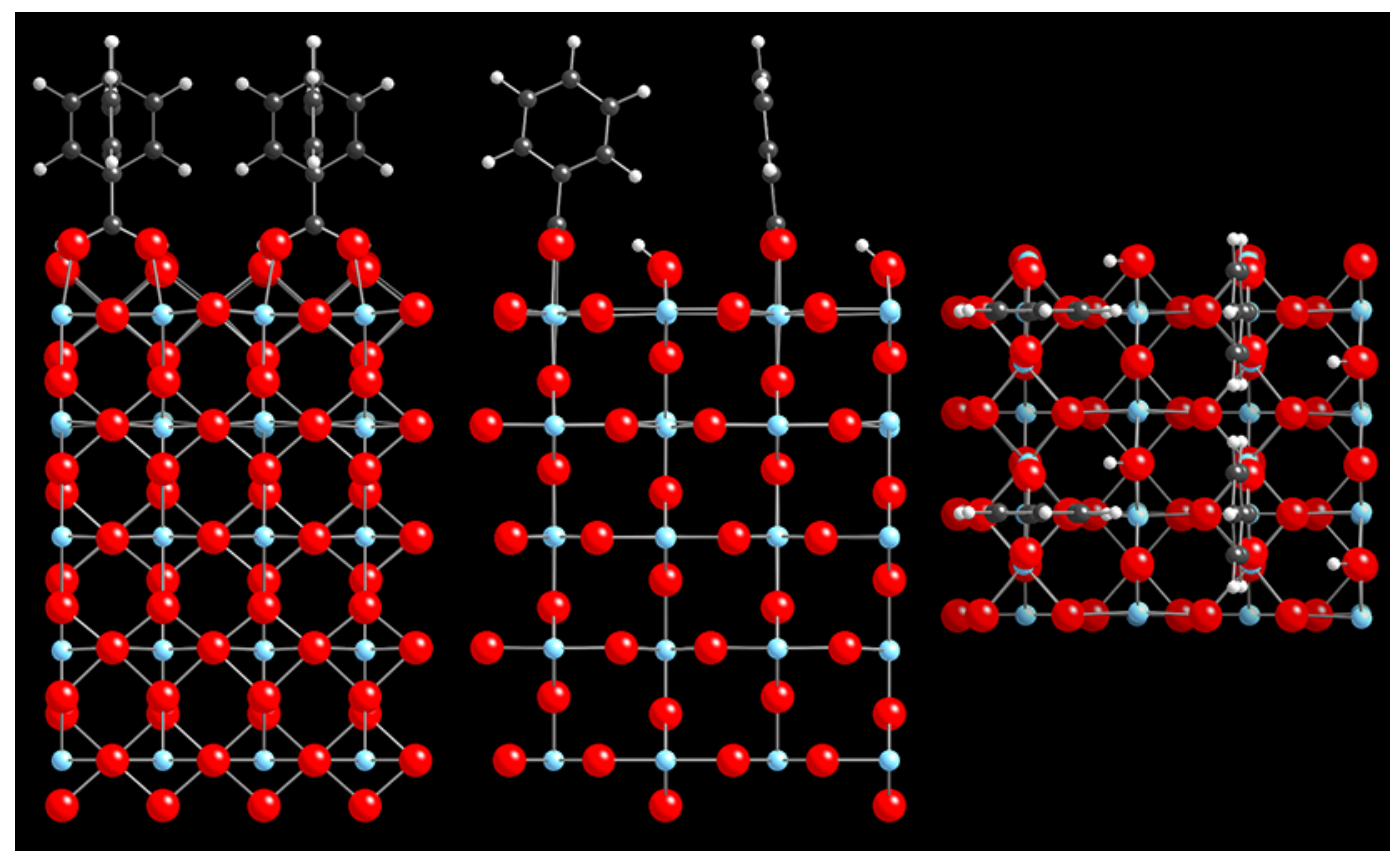

Figure S 5: Structure of the relaxed supercell used to represent the tilted $\mathrm{T}$ benzoate dimer. The views are from the side along the (left) [1 $\overline{10}$ ] and (middle) [001] directions, and from the (right) top. 


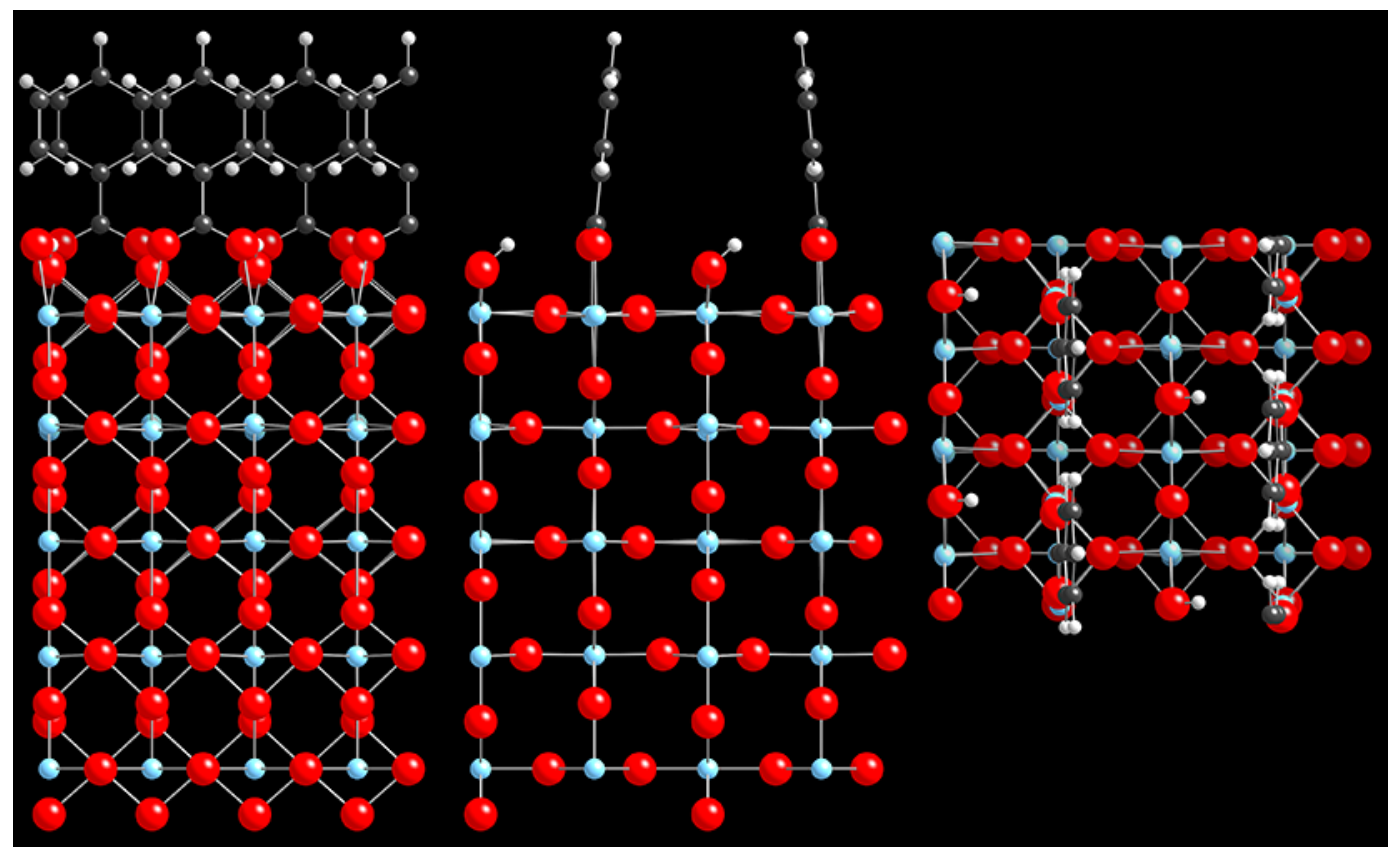

Figure S 6: Structure of the relaxed supercell used to represent the tilted staggered parallel benzoate dimer. The views are from the side along the (left) $[1 \overline{1} 0]$ and (middle) [001] directions, and from the (right) top.

\section{References}

${ }^{1}$ Wagner, C. D.; Davis, L. E.; Zeller, M. V.; Taylor, J. A.; Raymond, R. H.; Gale, L. H. Empirical Atomic Sensitivity Factors for Quantitative Analysis By Electron Spectroscopy for Chemical Analysis. Surf. Interface Anal. 1981, 3, 211-225.

${ }^{2}$ Fuentes, G. G.; Elizalde, E.; Yubero, F.; Sanz, J. M. Electron Inelastic Mean Free Path for Ti, TiC, TiN, and $\mathrm{TiO}_{2}$ as Determined By Quantitative Reflection Electron Energy-Loss Spectroscopy. Surf. Interface Anal. 2002, 33, 230-237.

${ }^{3}$ Kowalski, P. M.; Meyer, B.; Marx, D. Composition, Structure, and Stability of the Rutile $\mathrm{TiO}_{2}(110)$ Surface: Oxygen Depletion, Hydroxylation, Hydrogen Migration, and Water Adsorption. Phys. Rev. B 2009, 79, 115410 .

${ }^{4}$ Camellone, M. F.; Kowalski, P. M.; Marx, D. Ideal, Defective, and Gold-Promoted Rutile $\mathrm{TiO}_{2}(110)$ Surfaces Interacting With $\mathrm{CO}, \mathrm{H}_{2}$, and $\mathrm{H}_{2} \mathrm{O}$ : Structures, Energies, Thermodynamics, and Dynamics From PBE+U. Phys. Rev. B 2011, 84, 035413.

${ }^{5}$ Chabal, Y. J. Surface Infrared Spectroscopy. Surf. Sci. Reports 1988, 8, 211-357.

${ }^{6}$ Clark, I. T.; Aldinger, B. S.; Gupta, A.; Hines, M. A. Extracting Maximum Information From Polarized Surface Vibrational Spectra: Application to Etched, H-Terminated Si(110) Surfaces. J. Chem. Phys. 2008, $128,144711$. 